\title{
Some endangered plants producing edible fruits and seeds in Southeastern Nigeria
}

Apollos O.A. MereginI

Department of Forestry and Environmental Management, Michael Okpara University of Agriculture, Umudike, Abia State, Nigeria ago_dichi@yahoo.ca
* Correspondence and reprints

Fruits, 2005, vol. 60, p. $211-220$ (C) 2005 Cirad/EDP Sciences All rights reserved

DOI: 10.1051/fruits:2005028

RESUMEN ESPAÑOL, p. 220

\section{Some endangered plants producing edible fruits and seeds in Southeastern Nigeria.}

Abstract - Introduction. Although some of the non-formal food items consumed in Southeastern Nigeria are exotic, the indigenous products are becoming increasingly popular. There is also a growing interest among scientists in various disciplines to conduct research on nutritional, medicinal, industrial and other uses of the less studied and largely indigenous plants. Unlike the exotic fruits and seeds literature on the purely indigenous species is scanty. This paper is aimed at the documentation of some of the readily identified species producing fruits and seeds, which are considered to be endangered. Materials and methods. Selected plants producing edible fruits and seeds and considered to be endangered in Southeastern Nigeria were compiled in a list. Mature forms of the fruits and seeds were covered by means of color photographs and further categorized according to their families, growth habits, state of domestication, local names and methods of utilization. Results and discussion. Thirty plant species belonging to 19 families were recorded. The list includes 19 trees, 3 shrubs, 4 climbers and 2 rhizomatous monocots. About $27 \%$ of the species listed enjoy some degree of cultivation, while about $70 \%$ may still be found protected or wild. Up to $16 \%$ are still harvested only from the wild plants. The factors predisposing most of the species to extinction would be: loss of habitat due to deforestation and other forms of population pressure on the ecosystem; lack of interest in tree planting due to rather long gestation periods of existing genetic stock; increasing pressure and demand from few surviving stands; unsustainable harvesting methods. Conclusion. Ex situ conservation strategies, genetic and other tree improvement studies, encouragement of home garden and village forest settlement practices, and the introduction of incentives for tree planting are recommended strategies for conservation and sustainable production of the highly prized indigenous edible fruit and seeds in the area.

\section{Nigeria / endangered species / inventories / wild plants / domestication}

\section{Espèces produisant des fruits et des graines comestibles, menacées dans le sud-est du Nigéria.}

Résumé - Introduction. Bien que certaines plantes produisant des aliments non communs consommés dans le sud-est du Nigéria soient exotiques, les produits indigènes deviennent de plus en plus populaires. De plus, les scientifiques de diverses disciplines s'intéressent de plus en plus aux recherches sur les utilisations alimentaires, médicinales, industrielles et autres de plantes moins connues et, pour une grande part, indigènes. À la différence des fruits et des graines d'origine exotique, la littérature portant sur les espèces purement indigènes est pauvre. Cet article apporte des informations sur certaines espèces aisément identifiables, donnant des fruits et des graines consommés par les populations locales et considérées comme mises en danger. Matériel et méthodes. Des plantes à fruits et graines comestibles, considérées comme menacées au Nigéria, ont été listées. Des fruits et des graines mûrs ont été photographiés, puis classés par catégorie selon leurs familles, leur type de croissance, l'état de leur domestication, leurs noms vernaculaires et leur utilisation. Résultats et discussion. Trente espèces végétales réparties en 19 familles botaniques ont été répertoriées. La liste inclut 19 arbres, 3 arbustes, 4 plantes grimpantes et 2 plantes à rhizomes. Environ 27 \% des espèces recensées bénéficient de certaines techniques de culture, alors qu'environ $70 \%$ d'entre elles peuvent encore être trouvées protégées ou sauvages. Jusqu'à $16 \%$ sont encore récoltées sur des plants sauvages. Les facteurs prédisposant la plupart des espèces à l'extinction seraient : la perte d'habitat due au déboisement et à d'autres formes de pression des populations sur l'écosystème ; le manque d'intérêt pour la plantation d'arbres, du fait de la lenteur de mise en route des actions génétiques existantes; la pression et la demande croissantes de ces espèces issues d'un nombre restreint de sites où elles se maintiennent ; des méthodes de récolte non préconisées. Conclusion. Des stratégies de conservation ex situ, des études de génétiques, l'encouragement de pratiques pour l'établissement de jardins de case et de règlements d'exploitation de la forêt, ainsi que le lancement d'incitations pour la plantation d'arbres, sont des stratégies recommandées pour la conservation et la production durable de ces espèces fortement prisées dans la région d'étude.

Nigéria / espèce en danger / inventaire / plante sauvage / domestication 


\section{Introduction}

The feeding habits of the peoples of Southeastern Nigeria are dominated by the starchy foods of root and tuber origin usually taken with soups of various vegetables [1]. In addition to the bulky foods such as yam, cassava, cocoyam and plantains, peoples of Southeastern Nigeria consume a wide range of fruits and masticatories outside formal meal times such as during reception of visitors, opening of ceremonies, making of vows and pledges at marriages and while out in the fields or at other leisure times. Many of the fruits and seeds in this category, some of which serve as soup condiments, are often harvested from wild or protected plants. Many are often packaged as special gifts to friends and relations resident in cities within Nigeria and overseas. It may be necessary to stress that the demand for and consumption of the fruits and seeds cut across age brackets, standards of living and literacy levels. Thus, an account of the feeding habits of the peoples of Southeastern Nigeria will be incomplete without mention of these non-formal but vital food items.

Although some of the non-formal food items consumed in the area are exotic, the indigenous products are becoming increasingly popular. Because many of the indigenous items are obtained from the widely distributed plants, their gathering, distribution and marketing have created opportunities for employment of a number of rural and urban inhabitants, especially women. There is also a growing interest among scientists in various disciplines to conduct research on nutritional, medicinal, industrial and other uses of the less studied and largely indigenous plants. In 2000, Schippers [2] acknowledged the vague definition for vegetables and thus included green or fresh fruits as vegetables. The use of some green fruits as part of dessert with main dishes is largely allied to the traditional food habits. Fruits and seeds are freely consumed, independent of the main carbohydrate meals. They are also taken when ripe or mature.

In 1980, Okigbo [1] provided a distinction and prepared separate lists of food plants of Southeast Asian and South and Central American origin from indigenous food plants cul- tivated, protected or wild. Unlike the exotic fruits and seeds, which have received a great deal of horticultural attention, literature on the purely indigenous species is scanty.

This paper is aimed at the documentation, through pictures, of some of the readily identified fruits and seeds, which are considered to be endangered. A discussion is also provided on the factors which predispose the species to extinction.

\section{Materials and methods}

\subsection{The study area}

Southeastern Nigeria lies between lat. $4^{\circ} 20^{\prime} \mathrm{N}$ and $7^{\circ} 00^{\prime} \mathrm{N}$ and long. $5^{\circ} 25^{\prime} \mathrm{E}$ and $9^{\circ} 35^{\prime} \mathrm{E}$. It is bounded on the East by the Republic of Cameroon, on the South by the Atlantic Ocean, on the West by the River Niger and on the North by Kogi, Benue and Taraba States of Nigeria. The zone includes Enugu, Anambra, Imo, Abia, Ebonyi, Akwa Ibom, Bayelsa, Cross River and Rivers States. However, two tribes and two major languages, Ibo and Ibibio/Efik, predominate. The zone comprises the most densely populated states in the country with an average of about 0.19 ha per capita [3].

The mean maximum daily temperature is $27^{\circ} \mathrm{C}$, but the maximum temperature does not exceed $35^{\circ} \mathrm{C}$. The minimum daily temperature decreases from the coast towards the interior but it does not fall below $18^{\circ} \mathrm{C}$ [4]. Rainfall ranges from about $4338 \mathrm{~mm}$ per annum in Bonny in Niger Delta to about $1675 \mathrm{~mm}$ per annum in Nsukka up north.

Soils are very varied including shallow stony soils, young soils derived from deposited materials and hydromorphic soils. Soils in most areas are described as acidic and of low fertility status [5].

The primeval tropical rainforest vegetation has been replaced in most areas with what Hopkins described as farm-fallow and oil palm bush following centuries of deforestation and slash and burn agriculture [6]. Home gardens and village fruit tree forests containing several protected indigenous edible plants are common, particularly in the most densely populated states [7-9]. 


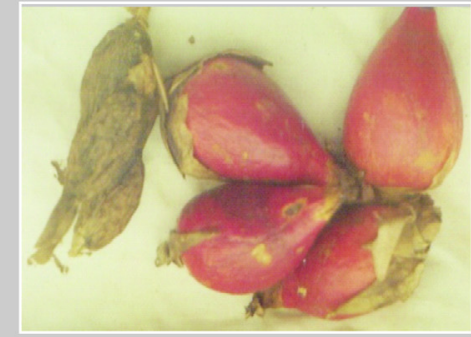

Aframomum melegueta K. Schum.

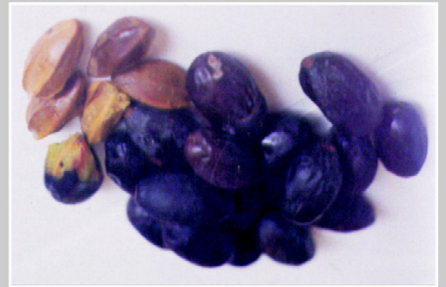

Canarium schweinfurhii Engl.

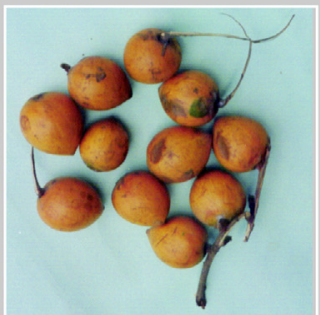

Chrysophyllum albidum G. Don

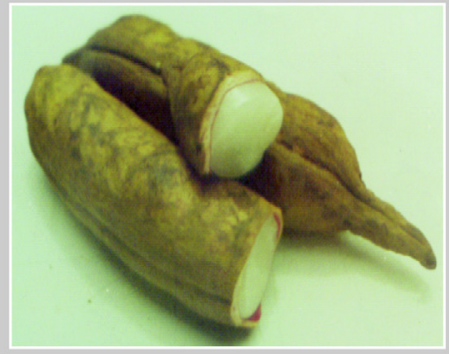

C. lepidota K. Schum

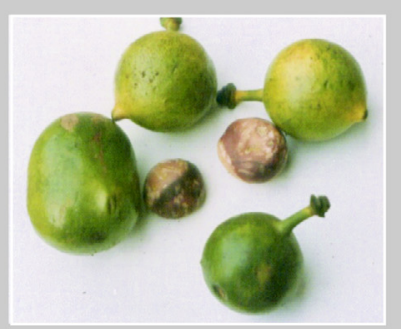

Buchholzia coriacea Engl.

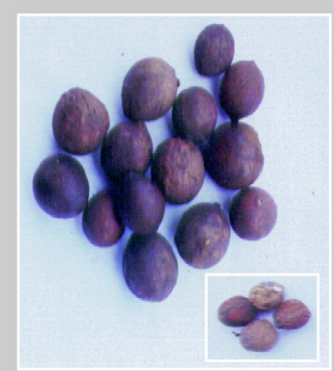

Coula edulis Baill.

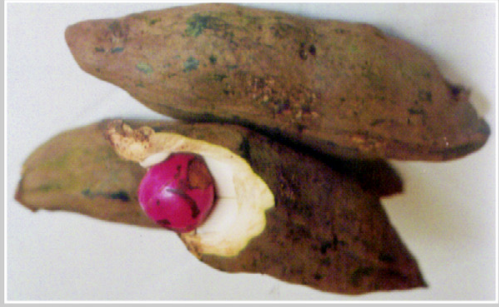

Cola acuminata Schott. \& Endl.

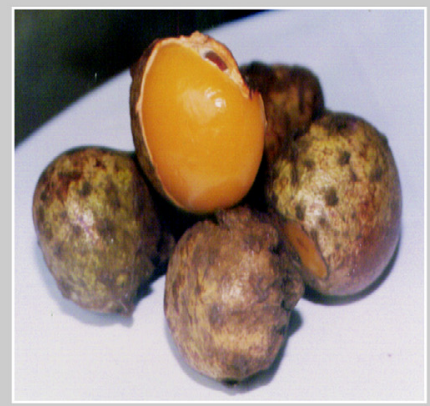

C. pachycarpa K. Schum.
Figure 1.

Some of the plant species producing edible fruits or seeds, found to be presently endangered in Nigeria (see colour pictures on Fruits' website).

\subsection{Listing of endangered species}

Mature or ripe fruits/seeds of plant species judged to be endangered in the area were collected and photographed (figures 1-4). A list of the plants was prepared, providing information on the scientific name, the family (sub-family in the case of leguminous 
Figure 2.

Some of the plant species producing edible fruits or seeds, found to be presently endangered in Nigeria (see colour pictures on Fruits' website).

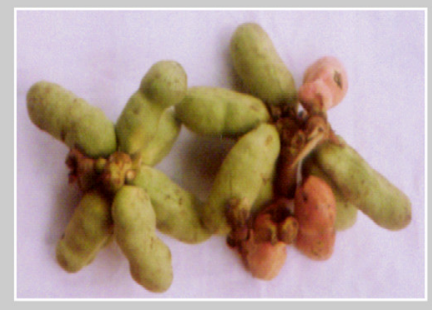

Denettia tripetala G. Bak.

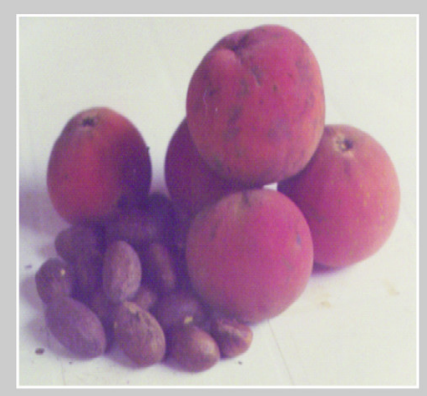

Garcinia cola Heckel

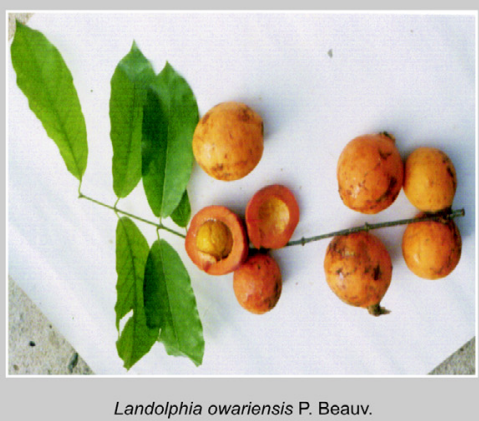

Landolphia owariensis P. Beauv.

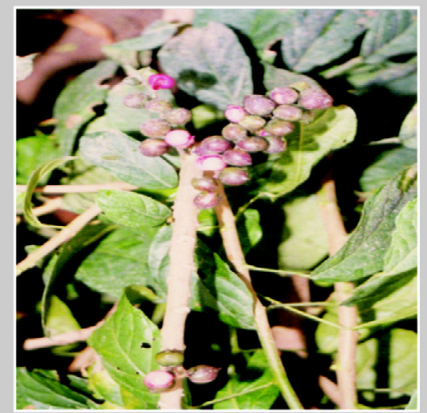

Maesobotrya sp. (Pax.) Hutch

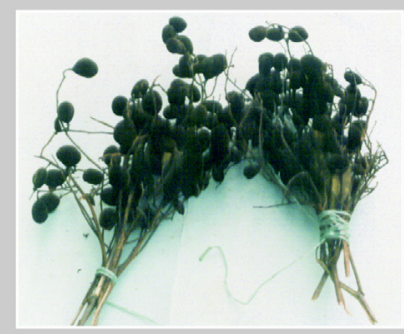

Dialium guineense Willd

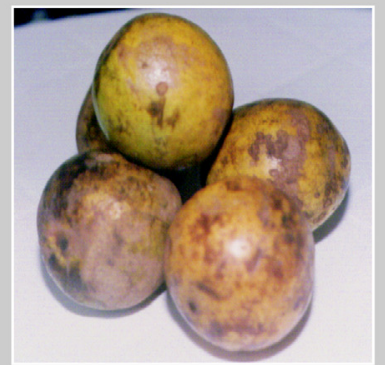

Irvingia gabonensis Baill.

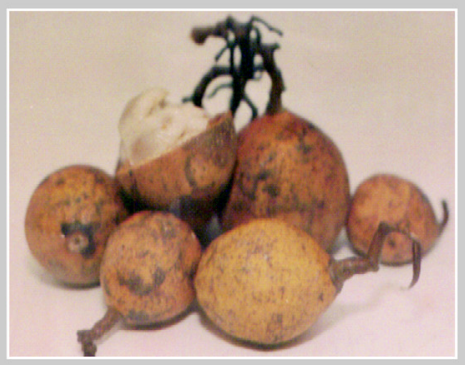

Landolphia sp.

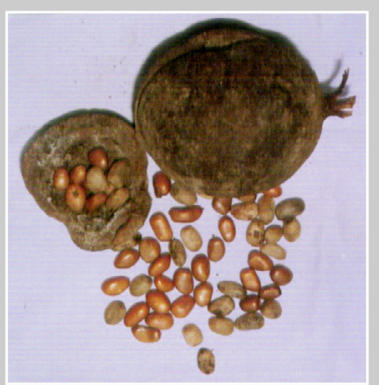

Monodora myristica (Gaertn.) Dunal species), growth habits, local names and a brief description of the parts commonly eaten. For each species, the degree of domestication was identified as protected, cultivated or wild. The common name was provided only in cases where the name is judged to be used fairly freely beyond the zone. Local flora [10-12] were freely referred to for proper classification and naming of the plant species. 


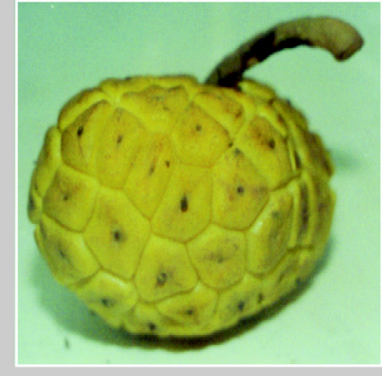

Myrianthus arboreus P. Beauv.

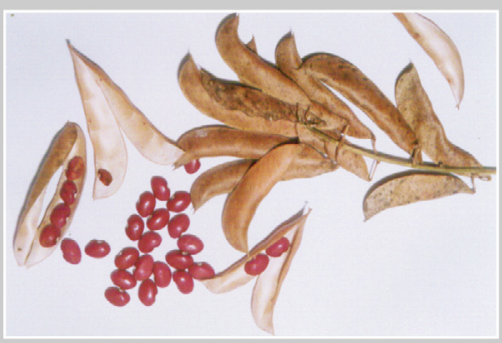

Phaseolus lunatus $\mathrm{L}$.

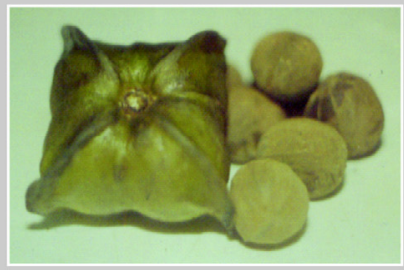

Plukenetia conophora Müll. Arg.

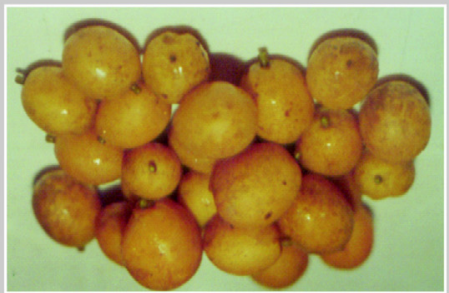

Spondias mombin L.

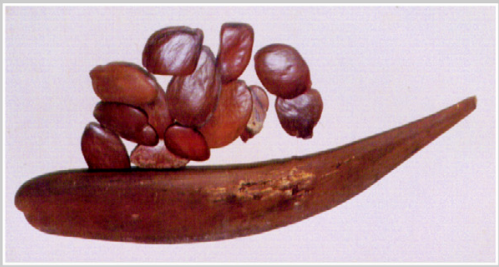

Pentaclethra macrophylla Benth.

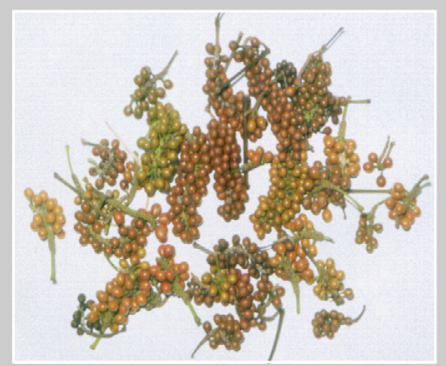

Piper guineense Schum. and Thonn.

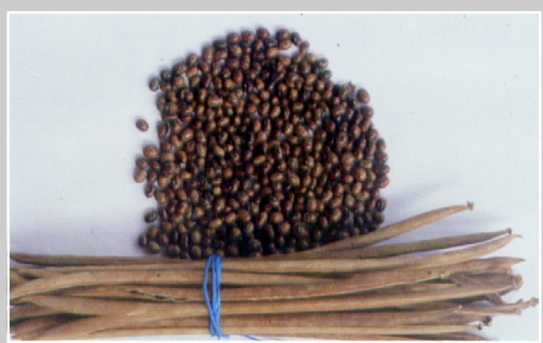

Sphenostylis stenocarpa Harms

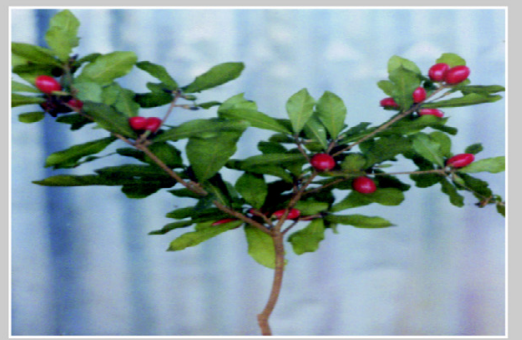

Synsepalum dulcificum Daniell.
Figure 3.

Some of the plant species producing edible fruits or seeds, found to be presently endangered in Nigeria (see colour pictures on Fruits' website).

\section{Results and discussion}

\subsection{Species listed}

Thirty plant species belonging to 19 different families were identified as endangered species (table I). Nineteen are trees while three are markedly shrubs. Four are climbing dicotyledons while two are rhizomatous monocots. About $27 \%$ of all the species listed have enjoyed some degree of domestication 
Figure 4.

Some of the plant species producing edible fruits or seeds, found to be presently endangered in Nigeria.

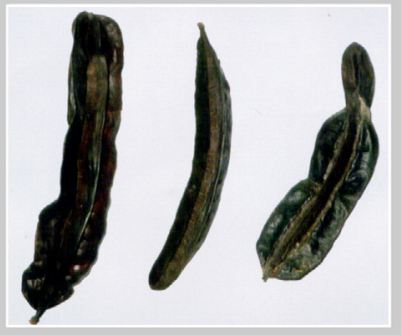

Tetrapleura tetraptera Taub.

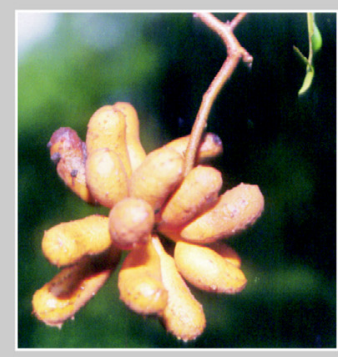

Uvaria chamae P .Beauv.

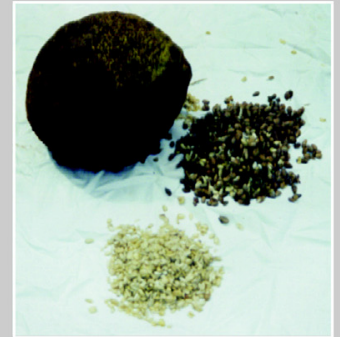

Treculia africana Decne

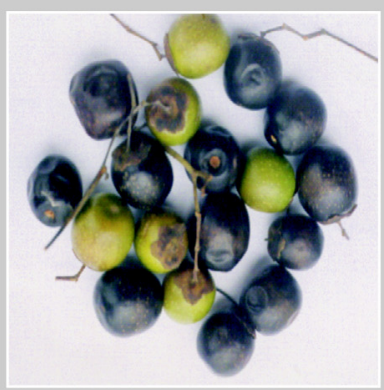

Vitex doniana Sweet
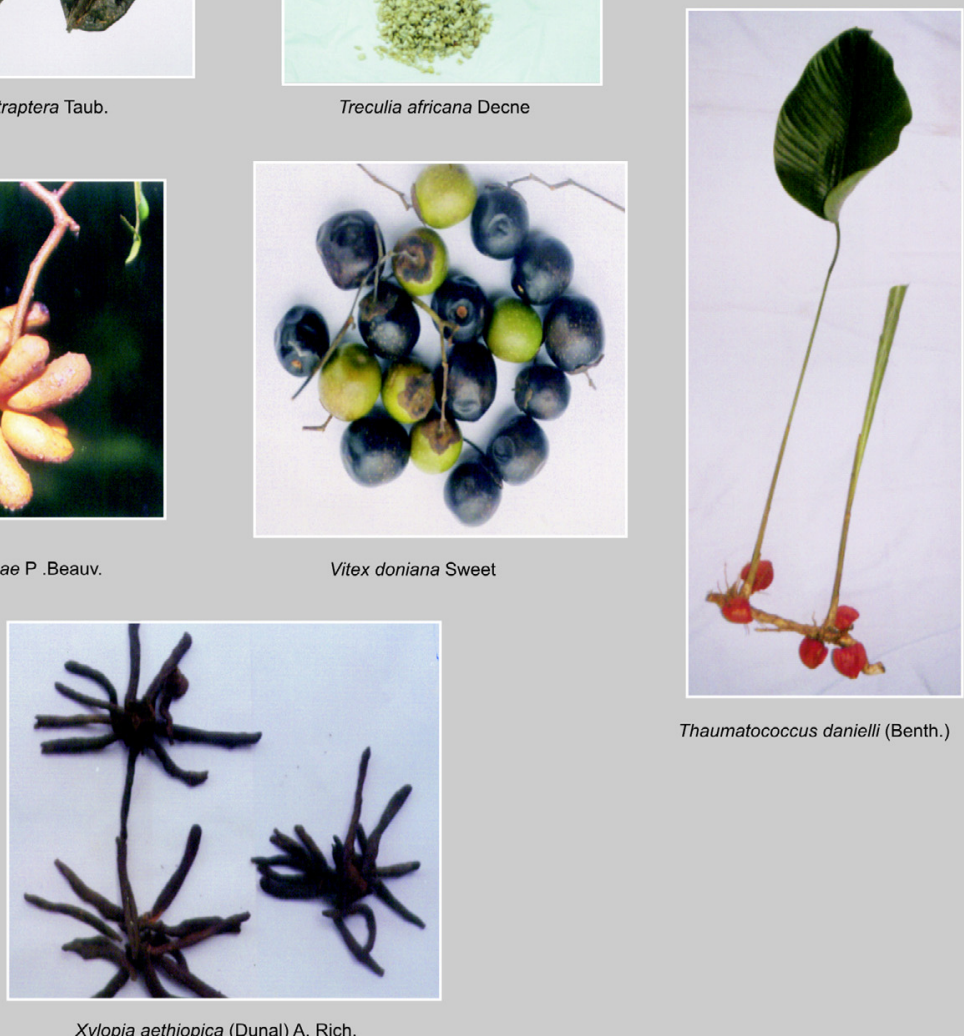

Thaumatococcus danielli (Benth.)

Xylopia aethiopica (Dunal) A. Rich and cultivation and 70\% may still be found protected or wild; about $16 \%$ of all the species are largely harvested from the wild.

\subsection{Concept of endangered species in the area}

Some edible plants of the climax vegetation of Southeastern Nigeria may have gone extinct through several years of forest degradation and vegetation change. Thus, only a fraction of these plants would be listed (table I).

In 1980, Okigbo compiled a list of 106 edible plants including leaf and fruit vegeta- bles, oils, pulses, spice roots and tubers. According to his results, thirty-one (29\%) are exotic and of Asian and South and Central American origin

Nearly all the fruit trees that are commonly planted in homes or in industrial plantations are the exotic species; they include Mangifera indica, Psidium guajava, Persea gratissima, Theobroma cacao and the Musa species.

The inclusion of any plant in the list of endangered species in southeastern Nigeria is based largely on the fact that little or no effort is made to propagate the indigenous 
Table I.

Some endangered trees producing edible fruits or seeds in Southeastern Nigeria (for domestication stage: C, cultivated; P, protected; W, wild).

\begin{tabular}{|c|c|c|c|c|c|}
\hline Species (domestication stage) & Family & Common name & Local names & $\begin{array}{l}\text { Growth } \\
\text { habits }\end{array}$ & Part eaten \\
\hline $\begin{array}{l}\text { Aframomum melegueta K. Schum. } \\
\text { (C P W) }\end{array}$ & Zingiberaceae & $\begin{array}{l}\text { Alligator pepper } \\
\text { Melegueta } \\
\text { pepper }\end{array}$ & $\begin{array}{c}\text { Ose oji (lbo) } \\
\text { Ntuenibokk (Ibibio) }\end{array}$ & $\begin{array}{l}\text { Rhizomatus } \\
\text { monocots }\end{array}$ & $\begin{array}{l}\text { Peppery seed chewed often } \\
\text { with cola }\end{array}$ \\
\hline Buchholzia coriacea Engl. (P W) & $\begin{array}{l}\text { Cappari- } \\
\text { daceae }\end{array}$ & Wonder cola & Uke (lbo) & $\begin{array}{l}\text { Tree up to } \\
20 \mathrm{~m}\end{array}$ & $\begin{array}{c}\text { Seed eaten after prolonged } \\
\text { boiling }\end{array}$ \\
\hline Canarium schweinfurhii Engl. (P W) & Burseraecae & Aiele & $\begin{array}{c}\text { Ubemgba or } \\
\text { Agbaka (Ibo) } \\
\text { Ebenetidok (lbibio) }\end{array}$ & $\begin{array}{l}\text { Tree up to } \\
35 \mathrm{~m}\end{array}$ & $\begin{array}{l}\text { Fruit pericarp eaten when } \\
\text { softened with hot water }\end{array}$ \\
\hline Coula edulis Baill. (P W) & Olacaceae & Walnuts & $\begin{array}{c}\text { Udi (lbo) } \\
\text { Ekom (Ibibio) }\end{array}$ & $\begin{array}{l}\text { Tree up to } \\
20 \mathrm{~m}\end{array}$ & $\begin{array}{l}\text { Oily seed eaten fresh as for } \\
\text { walnut }\end{array}$ \\
\hline $\begin{array}{l}\text { Chrysophyllum albidum G. Don (C P W) } \\
\text { Syn. Gambeya africana (A. DC) Pierre }\end{array}$ & Sapotaceae & Star apple & $\begin{array}{l}\text { Udara (Ibo) } \\
\text { Udara (Ibibio) }\end{array}$ & $\begin{array}{l}\text { Tree up to } \\
30 \mathrm{~m}\end{array}$ & Thick fleshy pulp eaten fresh \\
\hline Cola acuminata Schott. \& Endl. (P) & Sterculiaceae & Cola nut & $\begin{array}{c}\text { Oji (lbo) } \\
\text { lbong (Ibibio) }\end{array}$ & $\begin{array}{l}\text { Tree up to } \\
20 \mathrm{~m}\end{array}$ & Seed eaten fresh \\
\hline C. lepidota K. Schum. (P W) & Sterculiaceae & Monkey cola & $\begin{array}{l}\text { Ochicha (lbo) } \\
\text { Ndiya (Ibibio) }\end{array}$ & $\begin{array}{l}\text { Tree up to } \\
18 \mathrm{~m}\end{array}$ & White thick seed aril eaten fresh \\
\hline C. pachycarpa K. Schum. (P W) & Sterculiaceae & Cola & $\begin{array}{l}\text { Ochicha (Ibo) } \\
\text { Ndiya (Ibibio) }\end{array}$ & $\begin{array}{l}\text { Tree up to } \\
18 \mathrm{~m}\end{array}$ & $\begin{array}{c}\text { Yellow thick seed aril eaten } \\
\text { fresh }\end{array}$ \\
\hline Denettia tripetala G. Bak. (F C P) & Annonaceae & Pepper fruit & $\begin{array}{c}\text { Mmimi (lbo) } \\
\text { Nkarika (Ibibio) }\end{array}$ & $\begin{array}{l}\text { Small tree up } \\
\text { to } 10 \mathrm{~m}\end{array}$ & $\begin{array}{l}\text { Whole fruit green or ripe pink } \\
\text { eaten fresh }\end{array}$ \\
\hline Dialium guineense Willd. (W) & $\begin{array}{l}\text { Caes- } \\
\text { alpiniaceae }\end{array}$ & - & $\begin{array}{c}\text { Nchichi } \\
\text { Nkwaa (lbo) } \\
\text { Ukak (Ibibio) }\end{array}$ & $\begin{array}{l}\text { Small tree } \\
\text { often } \\
\text { shrubby }\end{array}$ & $\begin{array}{l}\text { Soft pink seed aril eaten } \\
\text { fresh }\end{array}$ \\
\hline Garcinia cola Heckel (P) & Guttiferae & Bitter cola & $\begin{array}{l}\text { Akilu/Akara (lbo) } \\
\text { Efiat (Ibibio) }\end{array}$ & $\begin{array}{l}\text { Tree up to } \\
18 \mathrm{~m}\end{array}$ & Seed eaten fresh \\
\hline Irvingia gabonensis Baill. (P W) & Irvingiaceae & Bush mango & $\begin{array}{l}\text { Ugiri, Upupa (Ibo) } \\
\text { Uyo (Ibibio) }\end{array}$ & $\begin{array}{l}\text { Tree up to } \\
20 \mathrm{~m}\end{array}$ & $\begin{array}{l}\text { Thick fleshy mesocarp eaten } \\
\text { fresh } \\
\text { Seed is soup condiment }\end{array}$ \\
\hline Landolphia owariensis P. Beauv. (P W) & Apocynaceae & $\begin{array}{l}\text { Landolphia } \\
\text { Madagascar } \\
\text { rubber- }\end{array}$ & $\begin{array}{c}\text { Utu (lbo) } \\
\text { Enwang (Ibibio) }\end{array}$ & $\begin{array}{l}\text { Woody } \\
\text { climber }\end{array}$ & Sour pulp in fruit eaten \\
\hline Landolphia sp. (P W) & Apocynaceae & Landolphia- & $\begin{array}{c}\text { Utuohia (lbo) } \\
\text { Ewang Ikot (lbibio) }\end{array}$ & $\begin{array}{l}\text { Woody } \\
\text { climber }\end{array}$ & Sweet pulp in fruit eaten \\
\hline Maesobotrya sp. (Pax.) Hutch (W) & $\begin{array}{l}\text { Euphor- } \\
\text { biaceae }\end{array}$ & - & $\begin{array}{l}\text { Uvune, Ubene } \\
\text { (lbo) } \\
\text { Nyata (Ibibio) }\end{array}$ & Shrub & $\begin{array}{l}\text { Juicy pulp around seeds eaten } \\
\text { fresh as in grapes }\end{array}$ \\
\hline Monodora myristica (Gaertn.) Dunal (P) & $\begin{array}{l}\text { Annonan- } \\
\text { aceae }\end{array}$ & African nutmeg & Ehiri, Ehuru & $\begin{array}{l}\text { Tree up to } \\
25 \mathrm{~m}\end{array}$ & $\begin{array}{l}\text { Seed used as spice or } \\
\text { condiment as in nutmeg }\end{array}$ \\
\hline Myrianthus arboreus P. Beauv. (W) & Moraceae & - & $\begin{array}{c}\text { Ujuju (Ibo) } \\
\text { Ndigo (Ibibio) }\end{array}$ & $\begin{array}{l}\text { Tree up to } \\
15 \mathrm{~m}\end{array}$ & $\begin{array}{l}\text { Portions of compound fruit } \\
\text { eaten fresh as in pineapple }\end{array}$ \\
\hline Pentaclethra macrophylla Benth. (C P W) & Mimosaceae & Oil bean & $\begin{array}{l}\text { Ugba (Ibo) } \\
\text { Ukana (Ibibio) }\end{array}$ & $\begin{array}{l}\text { Tree up to } \\
20 \mathrm{~m}\end{array}$ & $\begin{array}{l}\text { Seed boiled and shredded } \\
\text { eaten after some fermentation }\end{array}$ \\
\hline Phaseolus lunatus L. (C) & Fabaceae & Lima bean & Akidi (Ibo) & Twined & Boiled seed eaten \\
\hline Piper guineense Schum. and Thonn. (W) & Piperaceae & $\begin{array}{l}\text { African black } \\
\text { pepper }\end{array}$ & $\begin{array}{c}\text { Uziza } \\
\text { Adua (Ibibio) }\end{array}$ & $\begin{array}{l}\text { Clasping } \\
\text { climber }\end{array}$ & $\begin{array}{l}\text { Ripe seeds cooked in soups } \\
\text { for flavoring and as spice }\end{array}$ \\
\hline
\end{tabular}


Table I.

Continued.

\begin{tabular}{|c|c|c|c|c|c|}
\hline Species (domestication stage) & Family & Common name & Local names & $\begin{array}{l}\text { Growth } \\
\text { habits }\end{array}$ & Part eaten \\
\hline $\begin{array}{l}\text { Plukenetia conophora Müll. Arg. } \\
\text { (C P W) } \\
\text { Syn. Tetracarpidium conophorum }\end{array}$ & $\begin{array}{l}\text { Euphor- } \\
\text { biaceae }\end{array}$ & African walnut & $\begin{array}{c}\text { Ukpa (Ibo) } \\
\text { Ekporo (Ibibio) }\end{array}$ & $\begin{array}{l}\text { Woody } \\
\text { climber }\end{array}$ & Boiled seed eaten \\
\hline Sphenostylis stenocarpa Harms (C) & Fabaceae & African yam bean & $\begin{array}{l}\text { Odudu (Ibo) } \\
\text { Nsama (Ibibio) }\end{array}$ & Twined & Boiled seeds eaten \\
\hline Spondias mombin L. (P W) & $\begin{array}{l}\text { Anacar- } \\
\text { diaceae }\end{array}$ & $\begin{array}{l}\text { Jobo } \\
\text { Hog plum }\end{array}$ & $\begin{array}{l}\text { Uvuru (lbo) } \\
\text { Nsuka kara } \\
\text { (lbibio) }\end{array}$ & $\begin{array}{l}\text { Tree up to } \\
25 \mathrm{~m}\end{array}$ & Fruit pulp eaten as in plum \\
\hline $\begin{array}{l}\text { Synsepalum dulcificum Daniell. } \\
\text { (P W) }\end{array}$ & Sapotaceae & Miracle fruit & $\begin{array}{l}\text { Sotonso (lbo) } \\
\text { Nkpantung } \\
\text { (Ibibio) }\end{array}$ & Shrub & $\begin{array}{l}\text { Fruit with persistent sweet } \\
\text { taste } \\
\text { Sweetens sour drinks }\end{array}$ \\
\hline Tetrapleura tetraptera Taub. (P W) & Mimosaceae & Exotic spice & $\begin{array}{l}\text { Uhokiriho/Shoki- } \\
\text { risho (Ibo) } \\
\text { Edeminan (Ibibio) }\end{array}$ & $\begin{array}{c}\text { Tree up to } \\
25 \mathrm{~m}\end{array}$ & $\begin{array}{l}\text { Portions of fruit boiled in } \\
\text { soups as condiment } \\
\text { Charred fruit flavors drinking } \\
\text { water }\end{array}$ \\
\hline Treculia africana Decne (P C W) & Moraceae & African breadfruit & $\begin{array}{l}\text { Ukwa (Ibo) } \\
\text { Ediang (lbibio) }\end{array}$ & $\begin{array}{l}\text { Tree up to } \\
35 \mathrm{~m}\end{array}$ & $\begin{array}{l}\text { Shelled seeds boiled and } \\
\text { eaten as in rice }\end{array}$ \\
\hline $\begin{array}{l}\text { Thaumatococcus danielli (Benth.) } \\
\text { (P C W) }\end{array}$ & Marantaceae & Sweet prayer & Etere (Ibo) & $\begin{array}{l}\text { Rhizomatous } \\
\text { monocots }\end{array}$ & $\begin{array}{l}\text { Seed aril is sweet as in } \\
\text { miracle berry }\end{array}$ \\
\hline Uvaria chamae P. Beauv. (W) & Annonaceae & Finger root & $\begin{array}{l}\text { Mmimi-ohia (lbo) } \\
\text { Nkari Ikot (Ibibio) }\end{array}$ & $\begin{array}{l}\text { Straggling } \\
\text { shrub }\end{array}$ & Juicy seed aril eaten \\
\hline Vitex doniana Sweet (P W) & Verbenaceae & Black plum & Uchakoro (lbo) & $\begin{array}{l}\text { Tree up to } \\
15 \mathrm{~m}\end{array}$ & Ripe (black) fruit eaten fresh \\
\hline $\begin{array}{l}\text { Xylopia aethiopica (Dunal) } \\
\text { A. Rich. (P W) }\end{array}$ & Annonaceae & Negro pepper & $\begin{array}{c}\text { Uda (Ibo) } \\
\text { Atta (Ibibio) }\end{array}$ & $\begin{array}{l}\text { Tree up to } \\
20 \mathrm{~m}\end{array}$ & $\begin{array}{l}\text { Fruit pod cooked in soup for } \\
\text { flavoring and as spice }\end{array}$ \\
\hline
\end{tabular}

species in spite of the increasing demand for their products. Some factors would contribute to the predisposition of the species to extinction:

- The natural habitats of the plants, including the forests, and other stages of vegetation change are threatened by intensive agriculture, urban development and other infrastructure expansions.

- Traditional home gardens and village fruit tree forests, which serve as sanctuaries for in situ and some degree of ex situ conservation of the choice plants, are also threatened by back-to-back settlements and infrastructural development.

- Unlike the exotic species, which could fruit within 3 to 4 years after planting, most of the highly cherished fruit trees such as Chrysophyllum albidum, Denettia tripetala and Cola acuminata have very long gestation periods of 10 years or more. Thus, whereas people readily plant the former, there is a great deal of reluctance in planting the latter. Only two (Irvingia gabonenis and Treculia africana) out of the fruit trees listed have been subjected to some degree of improvement [13]. Through the process of grafting, the two species have been made to fruit within 4 years and at heights of less than 50\% of their natural habit. Nevertheless, the apathy over planting of indigenous fruits, including the improved species, appears to persist.

- As mentioned earlier, many of the fruit trees are highly cherished and there is a 
great deal of demand for the products by old and young people, including residents overseas. There is therefore tremendous pressure on existing protected or wild plants, some of which are wholly or partially cut to harvest the fruits.

Cola sp. seeds, particularly the local cola (C. acuminata), is a very important seed not only for hospitality in the reception of visitors but also for pledges and vows at marriages and other ceremonies in the area. The seed is highly symbolic among traditional chiefs. The chief will not normally accept or present any cola other than the seeds of C. acuminata. Thus, a single seed of this plant will cost (25 to 30) Nairas ( $1 \mathrm{~N}=$ $0.59 €)$, about five times the cost of very good seed of the exotic C. nitida. Our investigations have shown that a number of old C. acuminata trees in some locations stopped fruiting, probably due to changes in the prevailing environment.

\section{Conclusion and recommendations}

Peoples of Southeastern Nigeria depend on a wide range of edible indigenous fruits and seeds for staple foods, hospitality, chewing at leisure times, traditional pledges and vows and as a status symbol. Many of the species are not readily planted and are therefore in danger of extinction. Changes in the environment caused largely by human population pressure further predispose the protected and wild remnant species to total extinction, leading to loss of genetic resources, which they represent. In addition to the need for survey, categorization and documentation of the most socio-economically important species, there is an urgent need for the establishment of ex situ conservation centres for these species, particularly in relevant educational and research institutions.

The ex situ conservation efforts should be supported with genetic improvement and biotechnological studies to improve the growth habits of the trees concerned and select more desirable attributes of their products.
Government should support the education and enlightenment efforts on the importance of indigenous plant products with some policy of incentives such as tax rebate, free supply of propagation materials and land reforms to encourage the planting of the trees.

Government should discourage the proliferation of back-to-back housing estates, replacing these with settlements, which will provide a place for multistrata home gardens. Such settlements are associated with a range of sustainability indicators, including poverty alleviation, ecological stability, biodiversity maintenance and environmental soundness.

\section{References}

[1] Okigbo B.N., Plants and food in Igbo culture, Minist. Inf. Cult. Youths Sports, Ahiajoku lect., Owerri, Nigeria, 1980.

[2] Schippers R.R., African indigenous vegetables. An overview of the cultivated species, Natural Resources Institute / PCP EU Technical Centre for Agricultural and Rural Cooperation, Chatham, UK, 2000.

[3] Anon., Strategies for development of social forestry in Nigeria, Federal Department of Forestry (FDF), FORMECU/STAT, publ. no. 8 , Nigeria, 1985.

[4] Unamma R., Odurukwe S.O., Okereke H.S., Okoli O.O., Farming systems of Southeastern Nigeria, Natl. Root Crop. Res. Inst., Umudike, Nigeria, 1985.

[5] Agboola A.A., Soils of the Nigerian forest zone and their responses to cultivations, in: Okali D.U.U. (Ed.), Proc. MAB/UI workshop on the Nigerian rainforest ecosystem, Univ. Ibadan, Nigeria, 1979, pp. 80-94.

[6] Hopkins B., Forest and savanna, 2nd ed., Heineman Educ. Books, Ibadan London/ Ibadan, 1974, p. 54.

[7] Meregini A.O.A., Craige, Imo State plant species checklist, Report on survey of compound farms, Imo State, IITA, Ibadan, Nigeria, 1985.

[8] Okafor J.C., Fernandes E.C., Compound farms of Southeastern Nigeria. A predominant agroforestry home garden with small 
ruminant livestock, Agrofor. Syst. 5 (1987) 153-168.

[9] Okigbo B.N., Home gardens in the African humid tropics, in: Brazil and Marks (Eds.), Tropical Home Gardens, UNN/INRA, Accra, Ghana, 1990.

[10] Keay R.W.J., Trees of Nigeria, Clarendon Press, Oxford, UK, 1989.
[11] Hutchinson J., Dalziel J.M., Flora of west tropical Africa, Revised by Keay R.W.J., Crown Agents, London, UK, 1954.

[12] Burkill H.M., The useful plants of west tropical Africa, 2nd ed., Royal Botanic Gardens, Kew, UK, 1985.

[13] Okafor J.C., Improving edible species of forest products, Unasylva 5 (42) (1991) 17-33.

Especies que producen frutas y semillas comestibles, amenazadas en el sureste de Nigeria.

Resumen - Introducción. Aunque algunas plantas que producen alimentos no comunes consumidos en el sudeste de Nigeria sean exóticas, los productos indígenas se vuelven cada vez más populares. Además, los científicos de distintas disciplinas se interesan cada vez más en las investigaciones que se refieren a las utilizaciones alimenticias, medicinales e industriales entre otras, con plantas menos conocidas y, en gran parte, con plantas indígenas. A diferencia de los frutos y de las semillas de origen exótico, la literatura sobre especies puramente indígenas es escasa. Este artículo aporta información sobre algunas especies fácilmente identificables, que producen frutas y semillas consumidas por las poblaciones locales y que se consideran en peligro de extinción. Material y métodos. Se clasificaron algunas plantas de frutas y semillas comestibles, y que se consideran en situación de amenaza en Nigeria. Se fotografiaron algunas frutas y semillas maduras, luego se clasificaron por categoría según sus familias, su tipo de crecimiento, el estado de su domesticación, sus nombres vernáculos y su utilización. Resultados y discusión. Se enumeraron treinta especies vegetales distribuidas en 19 familias botánicas. La lista incluye 19 árboles, 3 arbustos, 4 plantas trepadoras y 2 plantas de rizomas. Alrededor del $27 \%$ de las especies contadas se benefician de algunas técnicas de cultivo, mientras que alrededor del $70 \%$ de ellas pueden aún encontrarse protegidas o salvajes. Hasta un 16\% aún se recogen sobre plantas silvestres. Los factores que predisponen a la mayoría de las especies para la extinción podrían ser: la pérdida del hábitat debido a la tala de árboles y a otras formas de presión de las poblaciones sobre el ecosistema; la falta de interés por la plantación de árboles, a causa de la lentitud de la puesta en marcha de las acciones genéticas existentes; la presión y la demanda creciente de estas especies resultantes de un limitado número de lugares donde se mantienen; métodos de cosecha no preconizados. Conclusión. Las estrategias de conservación ex situ, los estudios de genética, el estímulo de prácticas para el establecimiento de jardines familiares y de reglamentos de explotación del bosque, así como el lanzamiento de incentivos para la plantación de árboles, son estrategias recomendadas para la conservación y la producción sostenible de estas especies muy rebuscadas en la región de estudio.

Nigeria / especies en peligro de extinción / inventarios / plantas silvestres / domesticación 